\title{
Case management of malaria: Diagnosis
}

\author{
J Frean, ${ }^{1,2}$ MMed (Path Microbiol); B Poonsamy, ${ }^{1}$ MSc (Med); B Shandukani, ${ }^{3}$ BSc (Hons); D Moonasar, ${ }^{3} \mathrm{DrPh} ; \mathrm{J}$ Raman, ${ }^{4} \mathrm{PhD}$ \\ ${ }^{1}$ Centre for Opportunistic, Tropical and Hospital Infections, National Institute for Communicable Diseases, Johannesburg, South Africa \\ ${ }^{2}$ School of Pathology, University of the Witwatersrand, Johannesburg, South Africa \\ ${ }^{3}$ Malaria Directorate, National Department of Health, Pretoria, South Africa \\ ${ }^{4}$ Malaria Research Unit, South African Medical Research Council, Durban, South Africa
}

Corresponding author: J Frean (johnf@nicd.ac.za)

\begin{abstract}
Laboratory diagnosis of malaria in South Africa has traditionally relied on microscopic examination of stained blood films. More recently, rapid diagnostic tests (RDTs) have been introduced into routine use, and molecular methods like polymerase chain reaction are useful in certain situations. As with all laboratory tests, each technique has its advantages and disadvantages. Microscopy and RDTs, if appropriately quality assured, are adequate for clinical case management. For elimination, active surveillance will need to be expanded substantially, with wider use of more sensitive diagnostic nucleic acid amplification techniques, and/or serology. To facilitate surveillance activities, techniques suitable for field or near-field use would be ideal. A long-running external quality assessment programme in South Africa has shown some deficiencies in the quality of malaria diagnosis in routine laboratories. Quality systems across the spectrum of diagnostic facilities in South Africa need strengthening, to ensure progress towards elimination.
\end{abstract}

S Afr Med J 2013;103(10 Suppl 2):789-793. DOI:10.7196/SAMJ.7442

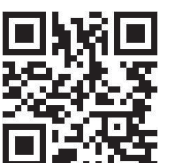

The prompt and accurate diagnosis of malaria is important because of its clinically non-specific nature and the potential for rapid deterioration into complicated and potentially fatal disease ${ }^{[1]}$ Apart from the obvious clinical benefit to the individual patient (i.e. in case management), accurate diagnosis is vital for surveillance in malaria control programmes for several reasons: in delineating local malaria epidemiology, identifying transmission 'hotspots', thereby triggering control programme action, and detecting gametocyte carriers, who may be minimally symptomatic. Additionally, studies of treatment or vaccine efficacy require accurate diagnosis, and misclassification (particularly false positives) can lead to major underestimation of efficacy or overestimation of resistance. ${ }^{[2,3]}$ The South African (SA) malaria treatment guidelines follow World Health Organization $(\mathrm{WHO})$ recommendations ${ }^{[4]}$ and explicitly require that a suspected malaria infection be confirmed or excluded with a blood test ${ }^{[5]}$ Unlike the scenario in much of Africa, transmission intensity in $\mathrm{SA}$ is too low to produce semi-immune populations, and the problem of distinguishing asymptomatic carriage of malaria parasites from clinical malaria does not generally apply to local-born populations, although it might affect recent immigrants from outside the borders. The latter situation has major implications for surveillance in the malaria endemic areas, given the porosity of SA borders, and the fact that most cases (around 70\%) of malaria in the country are imported from neighbouring states. ${ }^{[6-8]}$

\section{Diagnosis of malaria in SA}

Microscopic examination of Giemsa-stained films is still the operational gold standard for diagnosis of clinical malaria, albeit one widely acknowledged as imperfect. ${ }^{[9]}$ The sensitivity of thick blood film examination is limited by the training, skill and experience of the microscopist, the quality of the smear preparation and staining, and the level of the parasitaemia. As few as $5-50$ parasites/ $\mu \mathrm{l}$ can be detected by an expert, ${ }^{[0]}$ but many microscopists do not meet this standard. There are inherent technical problems that have long been recognised, such as parasites washing off during the thick film staining procedure (an estimated $60-90 \%$ of parasites are lost). ${ }^{[10]}$ Quantitative polymerase chain reaction (qPCR) has been used to measure this reduction (around 1 log i.e. 10 times) in parasite density. ${ }^{[11]}$ Microscopy equipment quality and maintenance, and staining quality and technique are often suboptimal, especially in remote, poorly-resourced settings. ${ }^{[12]}$ Quality of microscopic diagnosis can be highly variable. A study in a Mpumalanga Province malaria-risk area showed wide variation in positivity rates (6.3 - 45.8\%) of a set of 48 slides that was circulated among four diagnostic laboratories. ${ }^{[13]}$ In contrast, a legacy slide rechecking programme involving dedicated malaria programme microscopists in Limpopo Province claimed a $99.4 \%$ accuracy rate. ${ }^{[14]}$ Delays in hospital laboratory diagnosis were found during a review of malariarelated deaths in Mpumalanga Province. ${ }^{[15]}$ The present situation regarding quality assessment (QA) of blood film microscopy for case management in diagnostic laboratories is discussed later. HIV has a detrimental effect on malaria disease recognition and management, and this has been documented in the course of confidential enquiries into malaria-related deaths in SA ${ }^{[16]}$ The clinical threshold for malaria testing should be lowered in HIV-positive patients who present with fever in endemic areas. ${ }^{[16]}$

Previously, the SA provincial malaria control programmes undertook extensive active surveillance (case detection) in endemic areas, with control personnel sampling communities in which they were working at the time ${ }^{[13]}$ (P Kruger, personal communication). In many insecticide-sprayed areas, prevalence has dropped to such low levels that this is no longer widely done, and the inadequate sensitivity of microscopy or rapid diagnostic tests (RDTs) constrains their usefulness. The fact that malaria case investigators conducting active field surveillance cannot dispense the first-line antimalarial drug artemether-lumefantrine (as they could previously, when chloroquine fulfilled this role) has removed the motivation for on-site diagnosis and treatment. With better access to primary healthcare facilities than previously, most suspected cases are referred to the 
nearest clinic, where RDTs are generally available. If elimination is to be achieved, active surveillance practices will probably need to be revived and expanded to detect a sufficiently large enough pool of low-level infections to influence transmission. A corresponding move towards more sensitive detection methods will be required to detect the generally lower parasite loads expected. ${ }^{[17]}$

\section{Rapid antigen detection and molecular diagnostic methods}

A suitable alternative to microscopy in resource-deficient areas is the malaria RDT as it is relativity simple to perform, while allowing for point-of-care diagnosis and immediate malaria treatment. ${ }^{[18,19]}$ Malaria RDTs are lateral flow cassette-based immunochromatographic tests that detect specific Plasmodium antigens circulating in the peripheral blood of malaria-infected individuals. The binding of one of these antigens to dye-labelled antibodies results in the development of a visible coloured band in the test window of the RDT nitrocellulose strip, confirming the presence of malaria antigens. Sensitivity, specificity, stability and cost of a malaria RDT is greatly influenced by the choice of target antigen. ${ }^{[20]}$ Inherent disadvantages of RDTs for clinical case management are their inability to quantitate the parasite load and to track parasitological response to treatment.

\subsection{RDT use in SA}

In response to delayed as well as substandard malaria microscopic diagnosis, the National Department of Health $(\mathrm{NDoH})$ became the first African health ministry to implement a definitive malaria diagnosis policy using RDTs at all public sector health facilities in 1996. ${ }^{[21]}$ An RDT targeting the P. falciparum histidine-rich protein 2 (HRP2) was deployed, as P. falciparum infections accounted for over $90 \%$ of all confirmed malaria cases ${ }^{[22]}$ Field trials demonstrating a high degree of sensitivity and specificity, ${ }^{[23]}$ together with RDT userfriendliness, led to rapid acceptance of HRP2-based RDTs in the malaria endemic regions.

More efficient case management of non-malaria febrile patients and a more rational use of antimalarials in both Mpumalanga and Limpopo provinces followed RDT roll-out, a trend observed in other African countries. ${ }^{[24-27]}$ Unlike the other two malaria endemic provinces, RDT introduction in KwaZulu-Natal Province was associated with an increase in both case numbers ${ }^{[21]}$ and drug use. These increases were most likely fuelled by the emergence of sulphadoxine-pyrimethamine-resistant parasite populations in the region. ${ }^{[28]}$ Following a drug policy change in $2001,{ }^{[21]}$ RDTs ensured appropriate use of expensive artemisinin-based combination therapies and effective case management of non-malaria febrile cases in KwaZulu-Natal Province as well.

Since the initial deployment of RDTs in 1996, the parasite species structure has not altered substantially; with falciparum infections still the most prevalent in SA. In addition, field evaluations of HRP2based RDTs demonstrated a sustained high level of sensitivity with a marginal decline in specificity ${ }^{[29]}$ suggesting HRP2-based RDTs are still a viable option in SA. A survey of 102 public-sector laboratories in 2008 showed that 90 (88\%) were routinely using RDTs; this was up from $74 \%(85 / 115)$ in 2002 , and almost the same proportion $(131 / 147,89 \%)$ were using them in 2013 , reflecting the present fairly stable malaria situation in the country (National Institute for Communicable Diseases (NICD) data, unpublished). Investigation of malaria-related deaths suggested that in private medical practices, RDTs appear to be underused, and that education and training are required in diagnostic as well as other aspects of malaria case management in this sector. ${ }^{[16]}$

\subsection{Performance characteristics and quality of RDTs}

Although RDTs have proven to be extremely cost-effective in subSaharan Africa, ${ }^{[30]}$ their usefulness on the continent is sometimes constrained by sensitivity and specificity issues. Typical parasite loads in eliminating areas are expected to fall well below 100 parasites/ $\mu \mathrm{l}$ of blood, the approximate detection limit of RDTs, fuelling calls for the development and use of more sensitive nucleic acid-based techniques. ${ }^{[17]}$ This need, particularly in eliminating areas, is further highlighted when the HPR2-based RDT false-positivity rate is considered. Following successful malaria treatment, the HRP2 antigen persists in patients' blood for prolonged periods, resulting in false positives. ${ }^{[3]}$ There are also operator- and intrinsic test-related reasons for false positives. In a low-prevalence environment, this can translate into a very poor positive predictive value, an overestimation of malaria incidence and over-prescription of artemisinincontaining combination treatments (ACTs), and increased drug pressure. The replacement of HRP2-based RDTs with those targeting the P. falciparum lactate dehydrogenase ( $\mathrm{fLDH}$ ) antigen will not necessarily alleviate the false-positive issue. As both malaria gametocytes and asexual parasites produce $\mathrm{pfLDH}$, a RDT test may appear positive despite the clearance of symptom-causing asexual parasites. ${ }^{[32]}$ Sensitivity of HRP2-based RDTs can be further comprised by genetic modifications in the HRP2 gene. Sequence variations and deletions in the HRP2 and HRP3 genes affect HRP2based RDT sensitivity, ${ }^{[33]}$ most often resulting in false-negative results. At present these variations are relatively rare in Africa, but routine surveillance for these mutations is recommended to ensure RDT efficacy is sustained.

QA of RDT usage has lagged behind deployment in SA and there is currently no comprehensive quality-monitoring programme. Production lot variation and stability problems are known to occur, ${ }^{[34,35]}$ but at user level, routine quality control (QC) and proficiency testing (PT) are long-standing deficiencies. A study of proficiency among end-users of RDTs at primary healthcare level in Limpopo Province showed many problems of technique and interpretation, including false-positive and -negative rates of $4 \%$ and $15 \%$, respectively. ${ }^{[29]}$ A comprehensive quality programme would include regular QA/ QC of negative and positive RDTs from all health facilities using sensitive molecular methods like pooled PCR; batch testing of RDTs at a designated reference laboratory, prior to the delivery of RDTs to de-centralised health facilities, to ensure the minimum sensitivity and specificity is attained; regular refresher training on administering and interpreting RDT outcomes; and regular assessment of RDT transport and storage conditions at decentralised health facilities. Availability of positive control antigen material, long delayed because of technical production difficulties, appears to be imminent, and will help greatly in QA of RDTs and users. ${ }^{[36]}$ As an interim measure, PT using simulated samples ( $P$. falciparum in vitro culture medium) has been successfully trialled in routine laboratories in SA as part of an external QA programme (discussed later), but historically, malaria control programme personnel have not participated in this PT scheme.

\section{Priorities for diagnosis in the elimination strategy for SA}

Microscopy and RDTs, when performed and interpreted optimally, are suitable for case management and in the local context will need attention to quality improvement, maintenance and support, as discussed later. As SA embarks on its elimination agenda, mass screening and treating sufficient numbers of malaria carriers, whether symptomatic or asymptomatic, to interrupt malaria transmission 
becomes imperative. ${ }^{[37]}$ Detecting and eradicating every parasite, both asexual stages and gametocytes, using detection methods more sensitive to low-density infections, such as nucleic acid-based technologies, ${ }^{[17,38]}$ is therefore essential. Specificity of these methods also needs to be high to avoid false-positive results. Various suitable versions of DNA amplification, such as conventional and real-time qPCR, are readily available or already in use in the public and private sectors. PCR can also be used to monitor field RDT diagnostic quality. ${ }^{[39]}$ Loop-mediated isothermal amplification (LAMP) has potential field use, as technical requirements are much reduced in comparison with PCR. While an earlier version targeting ribosomal sequences was not very sensitive (detecting 100 parasites $/ \mu \mathrm{l}){ }^{[40]}$ performance characteristics of the latest formulation are comparable with nested PCR for clinical malaria diagnosis. ${ }^{[4]} \mathrm{A}$ preliminary field trial in Uganda showed good sensitivity and specificity in comparison with PCR for low-density infections. ${ }^{[42]}$ The technology is clearly potentially attractive for this purpose.

Very low levels of parasites are frequently or routinely missed by microscopy even under optimum conditions. ${ }^{[43]}$ As detecting and eradicating transmission foci are fundamental to malaria elimination, appropriate public sector laboratories should consider adopting techniques capable of identifying low-level gametocytaemia. A real-time quantitative nucleic acid sequence-based amplification (QT-NASBA) assay has been used successfully in Africa to determine sub-microscopic gametocyte carriage, ${ }^{[44]}$ but requires the collection of whole blood. In contrast, a reverse transcription PCR (RT-PCR) method can successfully conducted on fingerprick filter paper blood samples. ${ }^{[45]}$

An indirect approach to detecting malaria exposure is serological testing to detect parasite-specific antibodies. This technique has been used successfully in Somalia ${ }^{[46]}$ and Tanzania ${ }^{[47]}$ to determine malaria exposure and changes in transmission intensity. If SA is to meet its elimination target, then malaria confirmation using one of these more sensitive methods must become standard procedure at appropriate public sector laboratories. This will allow for more precise malaria case number calculations while assisting with control activity planning.

\section{Quality and training issues in malaria diagnosis}

In a National Malaria Control Programme review undertaken in 2009 , the uncertain state of quality assurance for malaria diagnostic tests, both for RDTs and microscopy, was identified as one of the weaknesses of the programme. ${ }^{[6]}$ Laboratory diagnosis of malaria in SA is carried out by the following groups:

- the National Health Laboratory Service (NHLS), which is the largest diagnostic pathology service in the country with over 260 laboratories, serving over $80 \%$ of the population;

- private laboratories, which serve the private sector; and

- provincial malaria control programmes, which actively investigate malaria cases and their contacts in the provinces in which malaria transmission occurs.

The first two groups are the best served in terms of quality assurance, because many of them comply with accreditation requirements that include participation in PT schemes. Despite this, when viewed collectively, many of these laboratories perform sub-optimally. Malaria control programme microscopists have not participated in these schemes, but there is other evidence that a substantial proportion do not meet minimum quality requirements (NICD, unpublished report).

\subsection{Personnel and training}

Microscopy of stained blood films is considered the gold standard for the routine laboratory diagnosis of malaria. ${ }^{[9,48,49]}$ Microscopy offers some advantages over RDTS, namely, the ability to detect the full malaria species spectrum, to quantitate the parasite burden (for prognostic value), and to serially monitor the parasitological response to treatment. The competency of microscopists is therefore crucial for accurate diagnosis. In SA, microscopists employed to perform malaria testing (often among other work) are usually medical technologists. Standards of training and proficiency of microscopists is highly variable. Malaria species identification is difficult, requiring expert training and much experience to master. Considering all these factors, and the findings from reviews of diagnostic quality ${ }^{[1,51,52]}$ leads one to conclude that, unsurprisingly, the quality of the results from our laboratories is inconsistent. The provincial malaria control programmes do not necessarily have strict qualification criteria in place for employment of microscopists and as a result their competence is even more variable, as recently assessed (see next paragraph) (NICD, unpublished report). Additional training is planned to address deficiencies at this level of the health service in the near future.

The NICD conducts training on diagnosis of blood parasites for routine laboratories, focusing on malaria. These training courses were previously limited to the NHLS laboratories in the central region of the country but from 2009 were extended to centres in all four NHLS regions. The main purpose of these courses is always practical microscopy but theory and practical components are also addressed. A limitation of this form of training is that there is no certainty that the course participants will return to their laboratories and implement the new techniques learnt, especially malaria identification. Some provincial malaria control programmes used to provide formal microscopist training, backed with regular refresher courses and a slide re-checking system to monitor quality, ${ }^{[14]}$ but this is no longer done. In 2012, at the request of the $\mathrm{NDoH}$, two refresher courses were run at the NICD specifically for control programme microscopists (NICD, unpublished report). Private laboratory groups include some malaria microscopy in the course of general laboratory training, including some refresher courses (J Trusler, personal communication). However, there are no dedicated malaria microscopists in these laboratories and they are not involved in active surveillance. Ideally, standardised training, competence assessment and certification, as recommended by the $\mathrm{WHO},{ }^{[53]}$ should be made available to the whole spectrum of laboratories, public and private, that are involved in malaria diagnosis.

\subsection{Quality assurance systems and accreditation}

In the past decade there has been a drive towards accreditation of medical laboratories. This process confirms that an adequate quality system is in place, to ensure accurate patient results. Accreditation ensures that laboratories use both internal and external QC. It also checks that adequate equipment and competent personnel are available. While the benefits of accreditation are numerous, the process to achieve accreditation is cumbersome and requires commitment from management and staff. With regard to public laboratories in our country, quality managers and coordinators have been recruited to implement and monitor quality systems in all laboratories. The process is ongoing, with regular audits conducted to check progress. The private laboratories follow a similar direction. The provincial malaria control programmes however, are lacking in this regard and require a great deal of support to strengthen their quality systems. Plans have been developed to support capacity 
strengthening of microscopists within malaria control programmes.

It is recommended that all blood films be examined by two microscopists; ${ }^{[48]}$ however, in instances where this is not possible more lenient criteria should be permitted. ${ }^{[54]}$ As a form of confirmation of results, some laboratories check all positive results microscopically; others confirm positives with a confirmatory test such as RDT, PCR or quantitative buffy coat (QBC) and some have no checks in place. As mentioned, centralised checking was done within the malaria control programmes in the past ${ }^{[14]}$ but is no longer done. It is evident that staff in many laboratories that use RDTs as a confirmatory test for microscopy tend to favour RDTs over microscopy, as they fail to understand the limitations of rapid tests. In some instances, stained blood films are not even viewed, a major quality deficiency in the system.

National guidelines stipulate that all laboratories that offer malaria testing in the country participate in a suitable external quality assessment (EQA) programme. ${ }^{[55]}$ In 1987, the South African Institute for Medical Research (predecessor of the NHLS blood parasites, which the NICD currently administers. ${ }^{[50-52]}$ This programme targets the SA public sector laboratories, but any laboratory may participate. The programme assesses laboratories' ability to correctly identify blood parasites (primarily malaria), to quantitate $P$. falciparum parasites on thin blood films, and occasionally to carry out relevant techniques such as slide staining or performing an RDT using a simulated blood sample. The national reference laboratory that conducts the programme and NICD) started an EQA programme for

belongs to an international EQA programme and is accredited to ISO standard 15189 (medical laboratory). The EQA programme was accredited to ISO standard 17043 (PT provider), by the South African National Accreditation System (SANAS) in early 2013. Participation in this programme by NHLS routine diagnostic laboratories is compulsory. There is also extensive voluntary participation by regional and private laboratories, but none from the malaria control programmes. Participation in such EQA programmes is not restricted to accredited laboratories and can be a key to identifying weaknesses in the quality systems of laboratories. ${ }^{[56]}$

A review of performance in the EQA programme over a 7-year period (Fig. 1) showed a $66 \%$ average rate of acceptable results (scores of 3 or 4 out of a maximum of 4 per challenge, i.e. $\geq 75 \%$ ), excluding the nonresponders. ${ }^{[51]}$ This means that on average, one third of laboratories are providing substandard malaria testing - not ideal for a country planning to eliminate malaria. There was wide variation in performance between surveys but unfortunately, no general trend towards improvement.

\section{Moving towards elimination}

To contribute to elimination of malaria in the country, three areas need to be addressed in terms of good quality laboratory diagnosis: $(i)$ standardisation of methods, (ii) introducing and maintaining good quality systems, and (iii) improving the competency of malaria microscopists and RDT users. The first aspect has been undertaken by the $\mathrm{NDoH}$, and has led the development of national quality guidelines for the laboratory diagnosis of

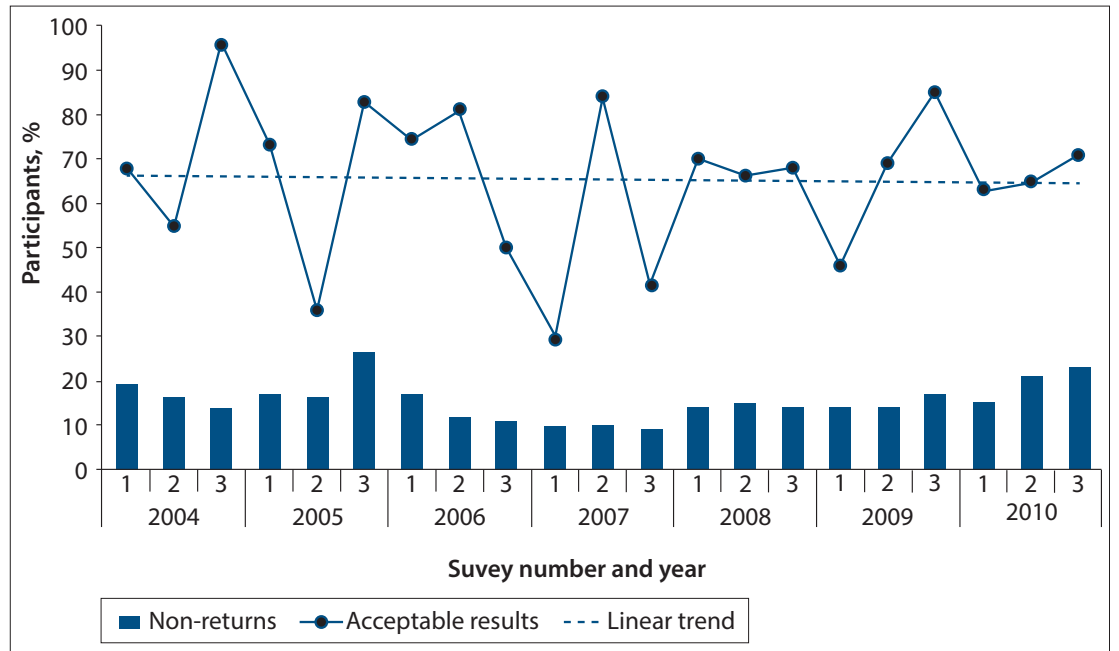

Fig. 1. Percentage of participants with acceptable results (scores $\geq 75 \%$ ) and non-returned results, per survey, in the Blood Parasite EQA Scheme, South Africa, $2004-20100^{[51]}$ malaria, ${ }^{[55]}$ soon to be deployed. The second aspect can be addressed by accreditation of all laboratories offering malaria training. Alternatively, quality officers could be employed to assist with monitoring of quality systems. Lastly, a solution to the competency problem would be to introduce a malaria microscopist certification programme.

\section{References}

1. Blumberg L, Baker L, Frean J. Clinical Management of Malaria. Pretoria: Foundation for Professional Development, 2006.

2. Ohrt C, Purnomo D, Sutamihardja A, Tang D, Kain KC. Impact of microscopy error on estimates of protective efficacy in malaria-prevention trials. J Infect Dis 2002;186:540-546. [http:// dx.doi.org/10.1086/341938]

3. O'Meara WP, Barcus M, Wongsrichanalai C, et al. Reader technique as a source of variability in determining malaria parasite density by microscopy. Malar J 2006;5:118. [http:// dx.doi.org/10.1186/1475-2875-5-118

4. World Health Organization Guidelines for the Treatment of Malaria. 2nd ed. Geneva: WHO, 2010. http://wwwwhoint/malaria/

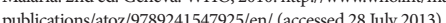

publications/atoz/978241547925/n/ (accessed 28 July 2013).

South African National Department of Health. Guidelines for the Tretment http://www.doh.gov.za/docs/policy/2011/malaria_treatment.
pdf (accessed 28 July 2013).

6. South African National Department of Health. Malaria diagnosis and case management. In: National Malaria Control Programme Review - 2009. Pretoria: $\mathrm{NDoH}, 2009$

7. Dube S, Ismail N, Hoosen AA. A retrospective review of malaria cases seen in a non-endemic area of South Africa. Travel Med Infect Dis 2008;6:296-300. [http://dx.doi.org/10.1016/j. tmaid.2008.06.010]

8. Weber IB, Baker L, Mnyaluza J, Matjila MJ, Barnes K, Blumberg L. The burden of imported malaria in Gauteng Province. S Afr Med J 2010;100:300-303.

9. Payne D. Use and limitations of light microscopy for diagnosing malaria at primary health care level. Bull World Health Organ 1988:66:621-626.

10. Dowling MAC Shute GT. A comparative study of thick and thin blood films in the diagnosis of scanty malaria parasitaemia. Bull World Health Organ 1966;34:249-267.

11. Bejon P, Andrews L, Hunt-Cooke A, Sanderson F, Gilbert SC, Hill AVS. Thick blood film examination for Plasmodium falciparum malaria has reduced sensitivity and underestimates parasite density. Malar J 2006;5:104. [http://dx.doi.org/10.1186/14752875-5-104]

12. Zikusooka CM, D McIntyre, KI Barnes. Should countries implementing an artemisinin-based combination malaria treatment policy also introduce rapid diagnostic tests? Malar J 2008;7:176. [http://dx.doi.org/10.1186/1475-2875-7-176]

13. Durrheim DN, Becker PJ, Billinghurst K, Brink A. Diagnostic disagreement - the lessons learnt from malaria diagnosis in Mpumalanga. S Afr Med J1997;87(5):609-611.

14. Hansford CH, van Vuuren CM. Diagnostic disagreement with Hansford CH, van Vuuren CM. Diagnostic disagreement with Durrheim DN, Frieremans S, Kruger P, Mabuza A, de Bruyn Durrheim DN, Frieremans $\mathrm{S}$, Kruger $\mathrm{P}$, Mabuza $\mathrm{A}$, de Bruyn
JC. Confidential inquiry into malaria deaths. Bull World Health Organ 1999;77(3):263-266

6. Mehta U, Durrheim DN, Blumberg L, et al. Malaria deaths as sentinel events to monitor healthcare delivery and antimalarial drug safety. Trop Med Int Health 2007;12(5):617-628. [http:// dx.doi.org/10.1111/j.1365-3156.2007.01823.x]

17. malERA Consultative Group on Diagnosis and Diagnostics. A research agenda for malaria eradication: Diagnoses and diagnostics. PLoS Med 2011;8(1):e1000396. [http://dx.doi. org/10.1371/journal.pmed.1000396]

18. Gitonga CW, Kihara JH, Njenga SM, et al. Use of rapid diagnostic tests in $\mathrm{CW}$, Khara $\mathrm{H}$, Njenga $\mathrm{SM}$, et al. Use of rapid diagnostic perforn malaria school perfor Med Hyg 2012,876) ajtmh.2012.12-0215

19. Moonasar D, Goga AE, Frean J Kruger P, Chandramohan D. An exploratory study of factors that affect the performance and usage of rapid diagnostic tests for malaria in the Limpopo Province, South Africa. Malar J 2007;6:74. [http://dx.doi. org/10.1186/1475-2875-6-74]

20. Kyabayinze DJ, Tibenderana JK, Odong GW, Rwakimari JB, Counihan $\mathrm{H}$. Operational accuracy and comparative persistent antigenicity of HRP2 rapid diagnostic tests for Plasmodium falciparum malaria in a hyperendemic region of Uganda. Malar I 2008;7:221. [http://dx.doi.org/10.1186/1475-2875-7-221]

21. Barnes KI, Durrheim DN, Little F, et al. Effect of artemetherlumefantrine policy and improved vector controct of artemetherlumefantrine policy and improved vector control on malaria burden in KwaZulu-Natal, South Africa. PLoS Med 2005;2(11):e330.
[http://dx.doi.org/10.1371/journal.pmed.0020330]

2. Moonasar D, Johnson CL, Maloba B, et al. Malaria. In: Ijumba P, Day C, Ntuli A, eds. South African Health Review 2003/0
Durban: South African Health Systems Trust, 2003:243-256. 
23. Durrheim DN, la Grange JJ, Govere J, Mngomezulu NM. Accuracy of a rapid immunochromatographic card test for Plasmodium falciparum in a malaria control programme in South Africa. Trans R Soc Trop Med Hyg 1998;92(1):32-33. [http://dx.doi.org/10.1016/S0035-9203(98)90944-0]

24. Chanda P, Hamainza B, Moonga HB, Chalwe V, Pagnoni F. Community case management of malaria using ACT and RDT in two districts in Zambia: Achieving high adherence to test results using community health workers. Malar J 2011;10:158. [http://dx.doi.org/10.1186/1475-2875-10-158]

25. Kunene S, Phillips AA, Gosling RD, Kandula D, Novotny JM. A national policy for malaria elimination in Swaziland: A first for sub-Saharan Africa. Malar J 2011;10:313. [http://dx.doi.org/10.1186/14752875-10-313]

26. Msellem MI, Mårtensson A, Rotllant G, et al. Influence of rapid malaria diagnostic tests on treatment and health outcome in fever patients, Zanzibar - A crossover validation study. PLoS Med 2009;6(4):e1000070. [http://dx.doi.org/10.1371/journal.pmed.1000070]

27. Mtove G, Hendriksen IC, Amos B, et al. Treatment guided by rapid diagnostic tests for malaria in Tanzanian children: Safety and alternative bacterial diagnoses. Malar J 2011;10:290. [http://dx.doi. org/10.1186/1475-2875-10-290]

28. Roper C, Pearce R, Bredenkamp B, et al. Antifolate antimalarial resistance in southeast Africa: A population-based analysis. Lancet 2003;361(9364):1174-1181. [http://dx.doi.org/10.1016/S01406736(03)12951-0]

29. Moonasar D, Goga AE, Kruger PS, et al. Field evaluation of a malarial rapid diagnostic test (ICT Pf). S Afr Med J 2009;99:810-813.

30. Shillcutt S, Morel C, Goodman C, et al. Cost-effectiveness of malaria diagnostic methods in subSaharan Africa in an era of combination therapy. Bull World Health Organ 2007;86:101-110. [http:// Saharan Africa in an era of combing
dx.doi.org/10.2471/BLT.07.042259]

31. Bell DR, Wilson DW, Martin LB. False-positive results of a Plasmodium falciparum histidinerich protein 2-detecting malaria rapid diagnostic test due to high sensitivity in a community with fluctuating low parasite density. Am I Trop Med Hyg 2005;73(1):199-203.

32. Wongsrichanalai C, Barcus MJ, Muth S, Sutamihardja A, Wernsdorfer WH. A review of malaria diagnostic tools: Microscopy and rapid diagnostic test (RDT). Am J Trop Med Hyg 2007;77(6 Suppl):119-127

33. Wurtz N, Fall B, Bui K, et al. Pfhrp2 and pfhrp3 polymorphisms in Plasmodium falciparum isolates from Dakar, Senegal: Impact on rapid malaria diagnostic tests. Malar J 2013;12:34. [http://dx.doi. org/10.1186/1475-2875-12-34

34. Bell D, Perkins MD. Making malaria testing relevant: Beyond test purchasing. Trans R Soc Trop Med Hyg 2008;102(11):1064-1066. [http://dx.doi.org/10.1016/j.trstmh.2008.05.007]

35. World Health Organization. The Use of Malaria Rapid Diagnostic Tests. Geneva: WHO, 2004. http:// www.who.int/malaria/publications/atoz/9290612045/en/ (accessed 28 July 2013).

36. Lon CT, Alcantara S, Luchavez J, Tsuyuoka R, Bell D. Positive control wells: A potential answer to remote-area quality assurance of malaria rapid diagnostic tests. Trans R Soc Trop Med Hyg 2005;99(7):493-498. [http://dx.doi.org/10.1016/j.trstmh.2005.01.001]

37. Rheeder JC, Targett GA, Shanks D, Greenwood B. Killing the Parasite. San Francisco: The Global Health Group, 2009:13.

38. McMorrow ML, Aidoo M, Kachur SP. Malaria rapid diagnostic tests in elimination settings - can they find the last parasite? Clin Microbiol Infect Dis 2011;17(11):1624-1631. [http://dx.doi.org/10.1111/ j.1469-0691.2011.03639.x]
39. Morris U, Aydin-Schmidt B, Shakerly D, et al. Rapid diagnostic tests for molecular surveillance of Plasmodium falciparum malaria - assessment of DNA extraction methods and field applicability. Malar J 2013;12:106. [http://dx.doi.org/10.1186/1475-2875-12-106]

40. Polley SD, Mori Y, Watson J, Perkins MD, et al. Mitochondrial DNA targets increase sensitivity of malaria detection using loop-mediated isothermal amplification. J Clin Microbiol 2010;48(8):28662871. [http://dx.doi.org/10.1128/JCM.00355-10]

41. Polley SD, González IJ, Mohamed D, et al. Clinical evaluation of a loop-mediated amplification kit for diagnosis of imported malaria. J Infect Dis 2013;208(4):637-644. [http://dx.doi.org/10.1093/infdis/jit183] 42. Hopkins $H$, González IJ, Polley SD, et al. Highly sensitive detection of malaria parasitemia in a malariaHopkins $\mathrm{H}$, González IJ, Polley $\mathrm{SD}$, et al. Highly sensitive detection of malaria parasitemia in a malaria-
endemic setting: Performance of a new loop-mediated isothermal amplification kit in a remote clinic endemic setting: Performance of a new loop-mediated isothermal amplification kit in
in Uganda. J Infect Dis 2013;208(4):645-652. [http://dx.doi.org/10.1093/infdis/jit184]

in Uganda. J Infect Dis 2013;208(4):645-652. [http://dx.doi.org/10.1093/infdis/jit184]
43. Okell LC, Ghani AC, Lyons E, Drakeley CJ. Submicroscopic infection in Plasmodium falciparum43. Okell LC, Ghani AC, Lyons E, Drakeley C). Submicroscopic infection in Plasmodium falciparumendemic populations: A systematic
[http://dx.doi.org/10.1086/644781]

44. Schneider P, Bousema T, Omar S, et al. (Sub)microscopic Plasmodium falciparum gametocytaemia in Kenyan children after treatment with sulphadoxine-pyrimethamine monotherapy or in combinatio with artesunate. Int J Parasitol 2006;36(4):403-408. [http://dx.doi.org/10.1016/j.jpara.2006.01.002]

45. Mlambo G, Vasquez Y, LeBlanc R, Sullivan D, Kumar N. Short report: A filter paper method for the detection of Plasmodium falciparum gametocytes by reverse transcription-polymerase chain reaction. Am J Trop Med Hyg 2008;78(1):114-116.

46. Bousema T, Youssef RM, Cook J, et al. Serologic markers for detecting malaria in areas of low endemicity, Somalia, 2008. Emerg Infect Dis 2010;16(3):392-399. [http://dx.doi.org/10.3201/ eid1603.090732]

47. Bousema T, Drakeley C, Gesase S, et al. Identification of hot spots of malaria transmission for targeted malaria control. J Infect Dis 2010;201(11):1764-1774. [http://dx.doi.org/10.1086/652456.]

48. Malaria Working Party British Committee for Standards in Haematology. The laboratory diagnosis of malaria. Clin Lab Haematol 1977;19(3):165-170. [http://dx.doi.org/10.1111/j.1365-2257.1997.tb00001.x]

49. Haditsch M. Quality and reliability of current malaria diagnostic methods. Travel Med Infect Dis 2004;2(3):149-160. [http://dx.doi.org/10.1016/j.tmaid.2004.03.001]

50. Dini L, Frean J, Stewart M. The South African parasitology quality assurance programme. Epidemiological Comments 2001;4:17-20.

51. Poonsamy B, Dini L, Frean J. Performance of clinical laboratories in South African parasitolog proficiency testing schemes between 2004 and 2010. J Clin Microbiol 2012;50(10):3356-3358. [http:// dx.doi.org/10.1128/JCM.01308-12]

52. Dini L, Frean J. Quality assessment of malaria laboratory diagnosis in South Africa. Trans R Soc Trop Med Hyg 2003;97(6):675-677. [http://dx.doi.org/10.1016/S0035-9203(03)80101-3]

53. World Health Organization. Universal Access to Malaria Diagnostic Testing An Operational Manual. Geneva: WHO, 2011. http://www.who.int/malaria/publications/atoz/9789241502092/en/ (accessed 28 July 2013).

54. Raghavan, K. Statistical considerations in the microscopical diagnosis of malaria, with special reference to the role of cross-checking. Bull World Health Organ 1966;34(5):788-791. [PMID: 5296237]

55. South African National Department of Health. National Malaria Diagnosis Quality Assurance Guidelines. Pretoria: NDoH (in press).

56. Badrick T. 2008. The quality control system. Clin Biochem Rev 2008;29(Suppl 1):S67-S70. 